\title{
Relationship between alcohol and nutrient intakes and blood status indices of older people living in the UK: further analysis of data from the National Diet and Nutrition Survey of people aged 65 years and over, 1994/5
}

\author{
CM Walmsley*, CJ Bates, A Prentice and TJ Cole \\ MRC Dunn Nutrition Unit, Downhams Lane, Milton Road, Cambridge CB4 IXJ, UK
}

Submitted 5 June 1998: Accepled 8 August 1998

\begin{abstract}
Objective: To examine the relationships between alcohol consumption and a range of nutrient intakes and blood status indices in older people.

Design: National Diet and Nutrition Survey: cross-sectional survey of nationally representative sample of people aged 65 years or over.

Setting: Mainland Britain during 1994/5.

Subjects: 1198 people ( 623 males, 575 females) aged 65 years or over, of whom 925 were living in private households and 273 were living in institutions.

Results: Intermediate alcohol consumption (particularly $0.1-14$ units week $^{-1} ; 1$ unit $=8 \mathrm{~g}$ ) derived from a 4-day diet diary or a 12 -month recall questionnaire, was associated with higher intakes of vitamins $C, E, B_{1}$, iron, calcium, energy from food, carbohydrate and non-starch polysaccharides than heavy alcohol consumption $\left(28+\right.$ units week $\left.{ }^{-1}\right)$ or abstinence, after adjustment for a number of factors (age, sex, domicile, social class, cigarette smoking, self-reported health, grip strength and total energy intake). Intermediate alcohol consumption was also associated with higher blood concentrations (independent of intake) of vitamin $C, \beta$-cryptoxanthin, lutein and calcium, with the lowest concentrations being found in heavy alcohol users. The lowest concentrations of serum ferritin were found in light drinkers and the highest levels in heavier alcohol drinkers. Alcohol consumption ranging from 0.1 to $>28$ units week ${ }^{-1}$ was directly correlated with intakes of $\mathrm{B}$ vitamins, total energy and fat, with blood concentrations (independent of intake) of lycopene, high density lipoprotein (HDL)-cholesterol, plasma pyridoxal phosphate and retinol, and with blood pressure and grip strength.

Conclusions: Compared with abstinence and heavy drinking, light to moderate alcohol consumption in older people is associated with higher intakes of certain nutrients, and higher blood concentrations (independent of intake) of some micronutrient status indices, including antioxidants. The explanation for the latter associations remains unclear and further investigation is recommended. Heavier alcohol consumption is associated with both beneficial and adverse effects with respect to nutrient intakes and health status.
\end{abstract}

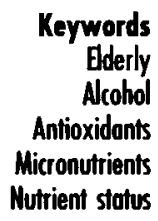

Adverse effects of excessive alcohol consumption and, more recently, beneficial effects of moderate alcohol consumption have been reported ${ }^{1-3}$.

Some of the adverse effects of alcohol on nutrient status may be due to an increased oxidative stress caused by ethanol toxicity, and a suboptimal antioxidant defence mechanism ${ }^{4-6}$; other effects may be due to malabsorption and increased urinary excretion of nutrients ${ }^{7-9}$, or poor dietary intakes ${ }^{10}$.

A number of studies in adult alcoholics without cirrhosis have reported low blood levels of antioxidants such as $\beta$-carotene ${ }^{11}, \alpha$-tocopherol, ascorbic acid ${ }^{5}$, selenium ${ }^{5,6}$ and other micronutrients such as plasma pyridoxal phosphate and folate ${ }^{12}$.

Conversely, a relationship between moderate alcohol consumption and a reduced risk of coronary heart disease has been observed in many epidemiological and experimental studies ${ }^{3,13}$. Evidence suggests that the relationship, which may be due partly to raised HDL-cholesterol, is causal, and the UK Department of Health currently advises men over 40 years and postmenopausal women of the benefits of drinking between 1 and 2 units $(8-16 \mathrm{~g})$ of alcohol a day ${ }^{3}$.

The relationships between alcohol, nutrition and 
health are of particular importance in older people who may be more vulnerable to nutrient deficiencies and health problems, due to decreasing food intakes ${ }^{14}$, impaired metabolism ${ }^{15}$, increased drugs usage and higher incidence of disease. In addition, the volume of total body water decreases in elderly people which results in a higher blood alcohol concentration for the same ingested amount of alcohol ${ }^{2,10}$.

There is little detailed information about alcohol use in relation to nutrition and health risk in older people. Some cross-sectional surveys of older people in Europe have found inverse relationships between alcohol intake and some nutrient status indices such as $\beta$-carotene ${ }^{16-18}$, but few studies have examined the relationships between intermediate alcohol intakes and micronutrient intakes and status in older people.

A cross-sectional National Diet and Nutrition Survey (NDNS) of people aged 65 years and over in mainland Britain was carried out during $1994 / 5$ and included assessment of food and nutrient intakes, a wide range of nutrient and other blood status indices, anthropometry and information on lifestyle, medicine usage and self-reported illness. This provided us with the opportunity to examine in detail the relationships between different categories of alcohol consumption and a range of antioxidant and other nutrient intakes and blood status indices in a large representative sample of older people.

\section{Subjects and methods}

This study used data from the National Diet and Nutrition Survey (NDNS) of people aged 65 years or over in mainland Britain to analyse further the relationships between consumption of alcohol and nutrient intakes and status of older people. Full details of the survey have been reported elsewhere ${ }^{19}$. The survey was carried out on behalf of the British government (Department of Health, and the Ministry of Agriculture, Fisheries and Food - MAFF) during the period October 1994 to September 1995. Social and Community Planning Research (SCPR) randomly selected a sample of older people, from 80 randomly selected postcode sectors within Britain. Both those living in private households (79\% of total) and in institutions i.e. residential and nursing homes (21\% of total) were represented in each sector. Predetermined numbers for statistical adequacy were recruited from both sexes and from each of the following agegroups: $65-74$ years; $75-84$ years and $85+$ years, and this required over-sampling of male subjects and of the older age groups. 2060 subjects participated in the survey, of whom $82 \%$ provided a full 4-day dietary record and $60 \%$ provided a blood sample.

A structured interview by trained field workers provided information on lifestyle, health, and eating and drinking habits of the participants. Participants were asked a series of questions regarding alcohol which included whether they currently drank alcohol (including drinks brewed or made at home), and for current drinkers, how often they had drunk different types of alcoholic drinks in the last 12 months and how much of each type they had drunk on any one day. From this information the subjects' alcohol intake in units per week was calculated by SCPR. Following the interview those living in private households were asked to keep a weighed record of all food and drink (including alcohol) consumed over 4 consecutive days. For those subjects who were unable or unwilling to keep a weighed record, a descriptive diary with household measures and portion sizes was kept by the participant or carer, and weights were assigned by the survey nutritionists. Those living in institutions were visited daily for 4 days by the interviewer who weighed one main meal on each visit. The participants or their carers were asked to keep a descriptive record of all food and drink consumed for the remainder of each 24 -hour period. The 4-day dietary records were coded and intakes of energy and nutrients (including alcohol) were calculated from the consumption data using a nutrient databank compiled by MAFF. A feasibility study in this age group had previously shown that there were no significant differences in average intakes of key nutrients between a 7-day or a 4-day dietary record, but levels of compliance and completion of the record were higher in those keeping the 4-day record ${ }^{20}$.

Anthropometric and grip strength measurements, information on the use of medications, blood pressure measurement and an early morning blood sample, usually fasting were obtained in the home by a trained nurse. The blood sample was taken after the 4-day diet record generally within a few days, and no more than 2 weeks later. Analyses for haematology and for haematinic nutrients (including serum folate and vitamin $\mathrm{B}_{12}$ ) were performed at Addenbrookes Haematology Laboratory, Cambridge, UK, and other biochemical index measurements were performed at the MRC Dunn Nutrition Unit, Cambridge, UK. The vitamin status index and other biochemical assay procedures are documented elsewhere ${ }^{29}$.

The analysis presented here was restricted to those 1198 participants who provided a full 4-day dietary diary and a blood sample. Alcohol intake in grams derived from the 4-day dietary record was converted into units of alcohol ( $8 \mathrm{~g}=1 \mathrm{unit}$ ), and then divided into weekly alcohol consumption categories as follows: $0,0.1-6.9,7-13.9,14-27.9$, and $28+$ units $^{\text {week }}{ }^{-1}$. Alcohol intake derived from the questionnaire was also divided into the same categories. To correct for the over-sampling a weighting variable was used and thus mean values (but not numbers of subjects) are 
based on a representative sample of people aged 65 years and over in mainland Britain. Participants who reported taking supplements of the nutrient in question during the dietary record were excluded from the analysis to prevent severe skewness of the data. Data reduction was performed using Microsoft Excel and data analysis was carried out using DataDesk computer programs (Data Description, Inc.). General linear models were used to perform linear tests for trends for which $P \leq 0.01$ was deemed to be statistically significant. Logistic regression and analysis of variance were used to test differences between means using the Scheffe test for which $P \leq 0.05$ was considered significant. Variables were transformed to normality where necessary, using either logarithmic, square root or reciprocal transformations. Means are presented after adjusting for the effects of age, sex and domicile (whether living in private households or institutions), and nutrient status indices are also adjusted for the corresponding nutrient intake. Results of further adjustment for social class, cigarette smoking, selfreported health and grip strength (B) are reported.

\section{Results}

Table 1 shows the number and proportion of older people in each category of alcohol intake derived by the two recording methods: a 4-day diet diary (which included alcohol in foods e.g. sherry trifle, as well as alcoholic drinks), and a 12 -month recall questionnaire (which included alcoholic drinks only). Results from the 4-day diary suggested that the majority of participants were non-drinkers, whereas the questionnaire indicated that the majority of participants were light drinkers $\left(<7\right.$ units week $\left.{ }^{-1}\right)$. Twenty percent of subjects from the 4-day diary, and $25 \%$ from the questionnaire consumed 7 or more units week ${ }^{-1}$, with $5 \%$ of these consuming 28 or more units week ${ }^{-1}$. Ten percent of subjects categorized as non-drinkers according to the questionnaire consumed some alcohol during the 4 days, but the average amount consumed was less than $1 \mathrm{gd}^{-1}$, and may have come from food. A fifth of subjects categorized as moderate drinkers (7-13.9 units week ${ }^{-1}$ ) according to the questionnaire did not drink during the 4 days. There was a Pearson correlation of $r=0.71$ between alcohol intake derived from the questionnaire and the diary, after adjustment for age, sex and domicile.

The analysis involved alcohol intake derived from both sources: but only those from the 4-day diary are given here. Where the results for intake derived from the questionnaire differ this is reported.

The association between group characteristics and alcohol intake is shown in Table 2 , adjusted for age, sex and domicile. The probability of drinking some alcohol was significantly greater in the 65-74 year olds than the $85+$ year olds, in males than females, and in those living in private households than those in institutions. More participants consumed alcohol in the non-manual classes than manual classes, those not receiving benefits (excluding pensions) than those who did, in January to March than July to September, in those with good health than those in bad health, those with less depression, and those not taking CNS or endocrine drugs than those taking these drugs. Of those who consumed alcohol, larger amounts were consumed by 65-74 year olds than those 75 years or over, by males than females, and by those living in private households (average $15 \mathrm{~g} \mathrm{day}^{-1}$ ) than in institutions (average $3 \mathrm{~g} \mathrm{day}^{-1}$ ), but after adjustment for age, sex and domicile, no differences between other group characteristics (listed in Table 2) and amount of alcohol (log transformed) consumed were observed (not presented). Alcohol categories derived from the questionnaire showed similar results, except that regarding drug usage the probability of consuming alcohol was greater only in those not taking cardiovascular drugs; and of those consuming alcohol, those from non-manual classes consumed more alcohol than manual classes (not presented).

Using logistic regression analysis it was observed that abstinence from alcohol was associated with those who reported that they had diabetes $(P=0.002)$, and with those who had suffered a stroke $(P=0.03)$, but abstinence was not associated with those who reported

Table 1 Proportion of participants in each category of alcohol intake*

\begin{tabular}{|c|c|c|c|c|c|}
\hline \multirow[b]{2}{*}{ Alcohol units $(\mathrm{g})$ week $^{-1}$} & \multicolumn{2}{|c|}{$\begin{array}{l}\text { Derived from a } \\
\text { 4-day diet diary }\end{array}$} & \multicolumn{3}{|c|}{ Derived from a 12-month recall questionnaire } \\
\hline & $\mathbf{n}$ & $\%$ & $\mathbf{n}$ & $\%$ & $\begin{array}{l}\% \text { not recording alcohol } \\
\text { during } 4 d \text { diet diary }\end{array}$ \\
\hline $\begin{array}{l}0(0) \\
0.1-6.9(0.1-55.9) \\
7-13.9(56-111.9) \\
14-27.9(112-223.9) \\
28 \text { or more }(224+)\end{array}$ & $\begin{array}{r}707 \\
253 \\
108 \\
69 \\
61\end{array}$ & $\begin{array}{r}59 \\
21 \\
9 \\
6 \\
5\end{array}$ & $\begin{array}{r}345 \\
535 \\
124 \\
105 \\
59\end{array}$ & $\begin{array}{r}30 \\
46 \\
11 \\
9 \\
5\end{array}$ & $\begin{array}{r}90 \\
63 \\
20 \\
14 \\
7\end{array}$ \\
\hline TOTAL & 1198 & 100 & 1168 & 100 & \\
\hline
\end{tabular}

-Excludes those without a full 4-day diary and those without a blood sample.

†1 unit $=8 \mathrm{~g}$ alcohol 
Table 2 Relationship between group characteristics and alcohol consumption*

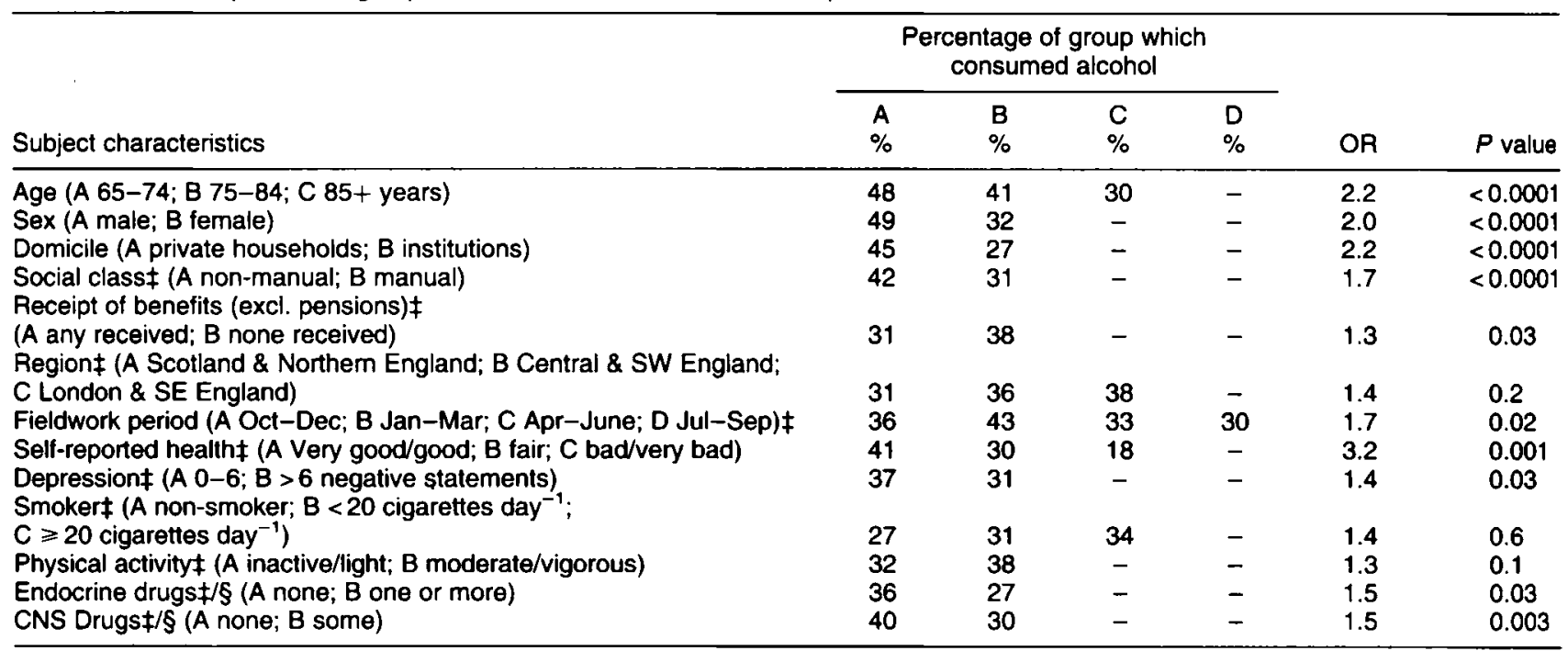

"Excludes those without a 4-day diet diary and those without a blood sample.

Weighted means and percentages are presented to correct for non-proportional sampling (see Subjects and methods section)

†Results obtained by logistic regression with alcohol (derived from a 4-day diary) as a binary dependent variable $(0=$ no alcohol; $1=$ alcohol).

$¥$ Adjusted for age category, sex and domicile.

§CNS (includes non-steroid anti-inflammatory drugs, opiates, drugs for anxiety, insomnia, schizophrenia, depression etc.). Endocrine drugs (includes drugs used in diabetes, thyroid and anti-thyroid drugs, corticosteroids). All other 11 drugs analysed (categories: gastrointestinal, cardiovascular, respiratory, antibiotics infection, cytotoxic, nutrition/blood, musculo-skeletal, skin, aspirin, eye) showed no significant associations with likelihood of alcohol consumption.

$\mathrm{OR}=$ odds ratio of highest versus lowest percentage within groups $A-D$.

NS, not significant if $P>0.05$.

that they had a long-standing illness in general, or with other self-reported specific illnesses such as cancer, mental illness, heart attack, hypertension, bronchitis, stomach ulcer or other digestive disorders, after adjustment for age, sex and domicile (not presented). After further adjustment for other factors (B), only diabetes remained significantly inversely associated with alcohol intake (derived from the 4-day diary $(P=0.02)$ and the questionnaire $(P=0.001))$.
Table 3 shows the amount of different types of alcoholic drinks consumed by total alcohol intake category, and the proportion who consumed the different types, adjusted for age, sex and domicile. As expected there was a highly significant direct correlation in amount of beer, wine, spirits and fortified wine consumed as total alcohol intake rises. The significant quadratic trend for fortified wine shows that those with intermediate total alcohol intakes drank

Table 3 Type of alcohol consumed as total alcohol intake increased*

\begin{tabular}{|c|c|c|c|c|c|c|c|}
\hline \multirow[b]{3}{*}{ Type of alcohol } & \multicolumn{4}{|c|}{ Alcohol intake (derived from 4-day diary) } & \multirow[b]{3}{*}{ Overall } & \multirow{2}{*}{\multicolumn{2}{|c|}{ Test for trend§ }} \\
\hline & \multirow{2}{*}{$\begin{array}{c}0.1-6.9 \\
\text { (units week }{ }^{-1} \text { ) }\end{array}$} & \multirow{2}{*}{$\begin{array}{c}7-13.9 \\
\text { (units week }\end{array}$} & \multirow{2}{*}{$\begin{array}{c}14-27.9 \\
\text { (units week }\end{array}$} & \multirow{2}{*}{$\begin{array}{c}28 \text { or more } \\
\text { (units week }{ }^{-1} \text { ) }\end{array}$} & & & \\
\hline & & & & & & Linear & Quadratic \\
\hline & & (g consump & n week $\left.{ }^{-1}\right) t$ & & & & \\
\hline $\begin{array}{l}\text { Beert } \\
\text { Wine } \dagger \\
\text { Spirits } † \\
\text { Liqueurs } \\
\text { Fortified wine } \\
\text { Cider and perry }\end{array}$ & $\begin{array}{c}\text { Mean(\%) } \\
484(27) \\
232(25) \\
60(21) \\
42(5) \\
110(20) \\
354(2)\end{array}$ & $\begin{array}{c}\text { Mean(\%) } \\
1355(30) \\
409(25) \\
144(26) \\
183(1) \\
290(14) \\
1187(4)\end{array}$ & $\begin{array}{c}\text { Mean(\%) } \\
2183(32) \\
531(23) \\
209(26) \\
44(3) \\
345(16) \\
0(0)\end{array}$ & $\begin{array}{c}\text { Mean(\%) } \\
4560(48) \\
1007(16) \\
577(29) \\
33(1) \\
279(5) \\
936(2)\end{array}$ & $\begin{array}{c}\text { Mean(\%) } \\
2000(33) \\
513(23) \\
229(25) \\
77(3) \\
314(15) \\
414(2)\end{array}$ & $\begin{array}{c}P \text { value } \\
<0.0001 \\
<0.0001 \\
<0.0001 \\
0.8 \\
<0.0001 \\
0.3\end{array}$ & $\begin{array}{l}P \text { value } \\
0.09 \\
0.9 \\
0.9 \\
0.1 \\
0.004 \\
0.9\end{array}$ \\
\hline $\begin{array}{l}\text { Total \%¥ } \\
n\end{array}$ & $\begin{array}{l}100 \\
205\end{array}$ & $\begin{array}{l}100 \\
161\end{array}$ & $\begin{array}{l}100 \\
132\end{array}$ & $\begin{array}{l}100 \\
107\end{array}$ & $\begin{array}{l}100 \\
605\end{array}$ & & \\
\hline
\end{tabular}

"Adjusted for age category, sex and domicile. Excludes those without a full 4-day diet diary, those without a blood sample, and those who did not consume alcohol. Weighted means are presented to correct for non-proportional sampling (see Subjects and methods section). Means obtained using analysis of variance.

tGeometric mean.

†Refers to \% of the total number in each alcohol intake category who consumed the different types of alcohol. $n$ is greater than the number of participants in each alcohol intake category as some participants consumed more than one type of drink.

$\S$ Test for trend of amount of different types of alcohol across the 4 alcohol intake categories.

NS, not significant if $P>0.01$.

1 unit $=8 \mathrm{~g}$ alcohol. 
relatively more fortified wine. As total alcohol intake increased the proportion of those drinking beer and spirits steadily increased while the proportion of those drinking wine and fortified wine decreased. More males than females drank beer ( $40 \%$ vs. $17 \%$ ), whereas more females drank wine (29\% vs $20 \%$ ) and fortified wine $(22 \%$ vs. $11 \%)$. Beer was drunk more in the manual classes, whereas wine was drunk more in the non-manual classes, in London and south east England, in those not receiving benefits (excluding pensions), those in good health and those with less depression (not presented).

Table 4 shows mean blood concentrations of a range of antioxidant, other micronutrient and lipid status indices in different alcohol intake categories adjusted for age, sex, domicile and intake of corresponding nutrient (including nutrient content of alcoholic drinks). Alcohol intake was directly correlated with plasma lycopene and HDL-cholesterol throughout the whole range of alcohol intake. The test for quadratic trends shows that the highest mean concentrations of plasma vitamin $C, \beta$-cryptoxanthin and serum folate were found in those with light to moderate alcohol intakes $\left(<14\right.$ units week $\left.{ }^{-1}\right)$ and the lowest concentrations were found in heavy alcohol consumers (28+ units week ${ }^{-1}$ ). (When nutrient intake was not adjusted for these associations remained significant, but in addition there was a direct correlation between alcohol intake and plasma pyridoxal phosphate (PLP) $(P \leq 0.0001)$ and a significant quadratic trend for red cell folate $(\mathrm{RCF})(P=0.01)$ showing higher concentrations in those with intermediate alcohol intakes (not presented).) After adjustment for age, sex, domicile, nutrient intake and other factors (B), significantly lower concentrations of serum ferritin were found in those with light $\left(<7\right.$ units week $^{-1}$ ) alcohol intakes with the highest intakes being observed in heavy drinkers, and the strength of the association between alcohol and $\beta$-cryptoxanthin and serum folate was reduced to $P=0.02$ and $P=0.03$ respectively. Alcohol intake derived from the questionnaire (not presented) showed a direct association with plasma lycopene, PLP, plasma retinol and HDL-cholesterol. Relatively higher concentrations of plasma $\beta$-cryptoxanthin, lutein and calcium were found at intermediate alcohol intakes, and the lowest concentrations were found in heavy drinkers, after adjustment for age, sex, domicile, nutrient intake and other factors (B).

Table 5 shows mean nutrient intakes (including nutrient content of alcoholic drinks) in different alcohol intake categories after adjusting for age, sex and domicile. Alcohol intake was directly correlated with intakes of vitamin $B_{2}, B_{6}$, retinol, folate, vitamin $B_{12}$, total energy and fat. The quadratic trends show relatively higher intakes of vitamins $\mathrm{C}, \mathrm{E}, \mathrm{B}_{1}$, iron, calcium, energy from food, carbohydrate and non-starch polysaccharides in those with intermediate alcohol intakes than in nondrinkers or heavy alcohol consumers. After further adjustment for other factors (B) the association between alcohol and retinol intake disappeared. Intake of vitamin $\mathrm{B}_{6}$ was directly correlated with alcohol intake in beer/ lager and spirit drinkers but not in wine drinkers, and folate intake was directly correlated in beer/lager drinkers but not in wine or spirit drinkers, after adjustment for age, sex, domicile and other factors (B) (not presented). Alcohol intake derived from the questionnaire (not presented) showed fewer associations with nutrient intake: after adjustment for age, sex, domicile and other factors (B), vitamin $B_{2}, B_{6}, B_{12}$, folate and total energy intake were directly correlated with alcohol. Relatively higher intakes of calcium, energy from food, carbohydrate and NSP were found in those with intermediate alcohol intakes.

When total energy intake, in addition to age, sex, domicile and other factors (B) was adjusted for, the significance of the association between alcohol and intake of vitamin $\mathrm{B}_{2}$ fell outside the $1 \%$ level $(P=0.2$ when alcohol was derived by 4-day diary; $P=0.06$ when derived from questionnaire). The significance of the association between alcohol (derived from the questionnaire only) and plasma lycopene was reduced when also adjusting for total energy intake $(P=0.045)$ : no other significant associations between alcohol and nutrient intakes and status were lost by the further adjustment for total energy intake.

Alcohol consumers as a whole were slightly more likely to consume meat products (bacon, sausages, burgers, ham, kebabs), pies and pasties than nondrinkers $(93 \%$ vs $90 \%$; odds ratio $1.5 ; P=0.04)$, more red meat and poultry were consumed by alcohol drinkers than non-drinkers $(P=0.03)$, and more fruit was consumed by those with intermediate alcohol intakes than non-drinkers or heavy drinkers $(P=0.002)$, after adjustment for age, sex, domicile and other factors (B). Alcohol consumption was not associated with any other of the following different food groups: white bread, brown bread, wholegrain and high-fibre breakfast cereal, other breakfast cereal, whole milk, semiskimmed milk, skimmed milk, cheese, fish and vegetables (not presented). Alcohol intake derived from the questionnaire showed only an association with fruit consumption: the likelihood of consuming fruit and amount of fruit consumed was greater in alcohol consumers, particularly those with intermediate alcohol intakes.

Table 6 shows the association of alcohol with other factors associated with health, after adjusting for age, sex and domicile. Alcohol intake was inversely associated with alkaline phosphatase and directly correlated with demispan, grip strength and haemoglobin. Lower levels of total plasma homocysteine (tHcy) and $\gamma$-glutamyl transferase (GGT) were found in those 


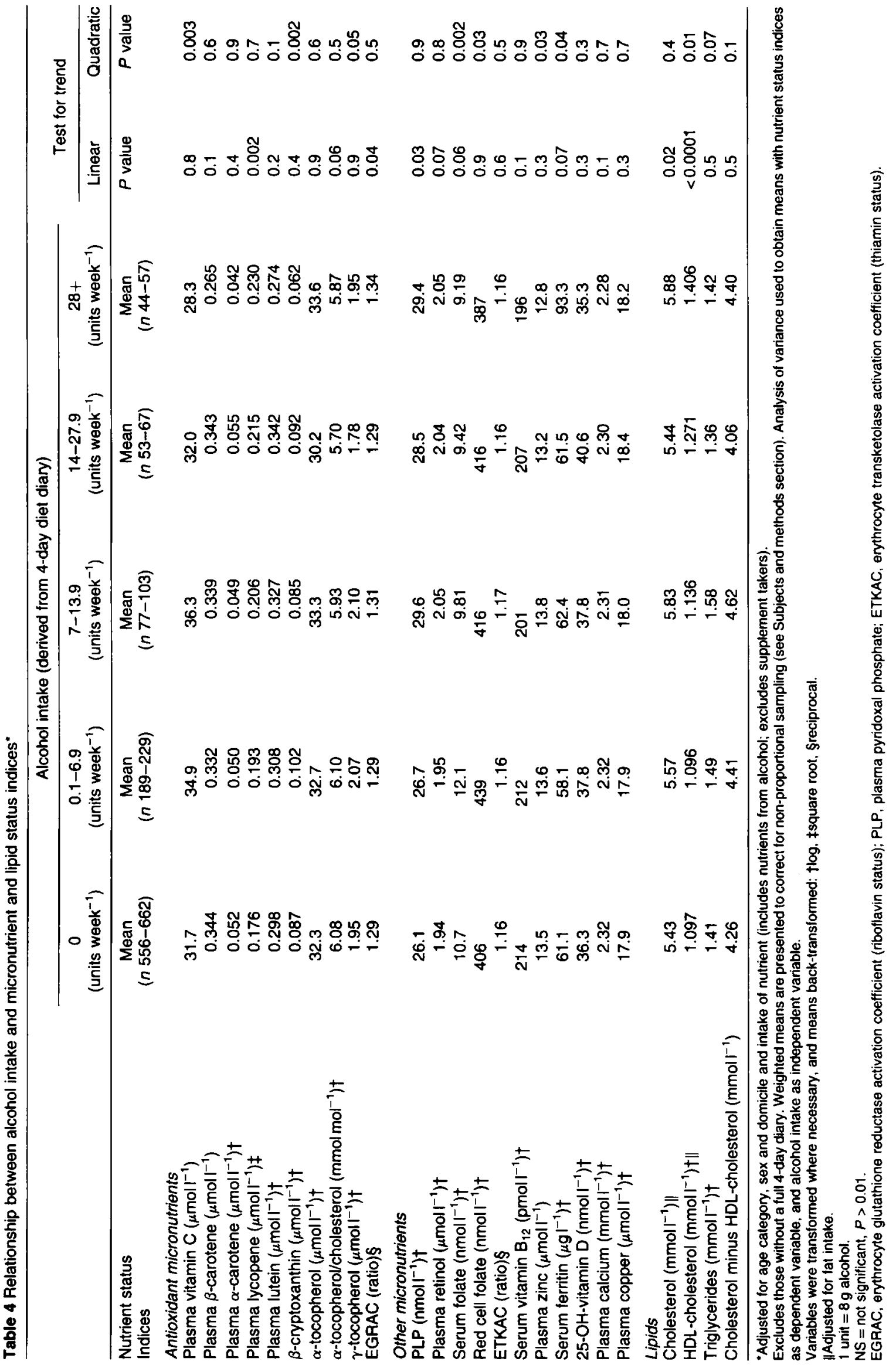




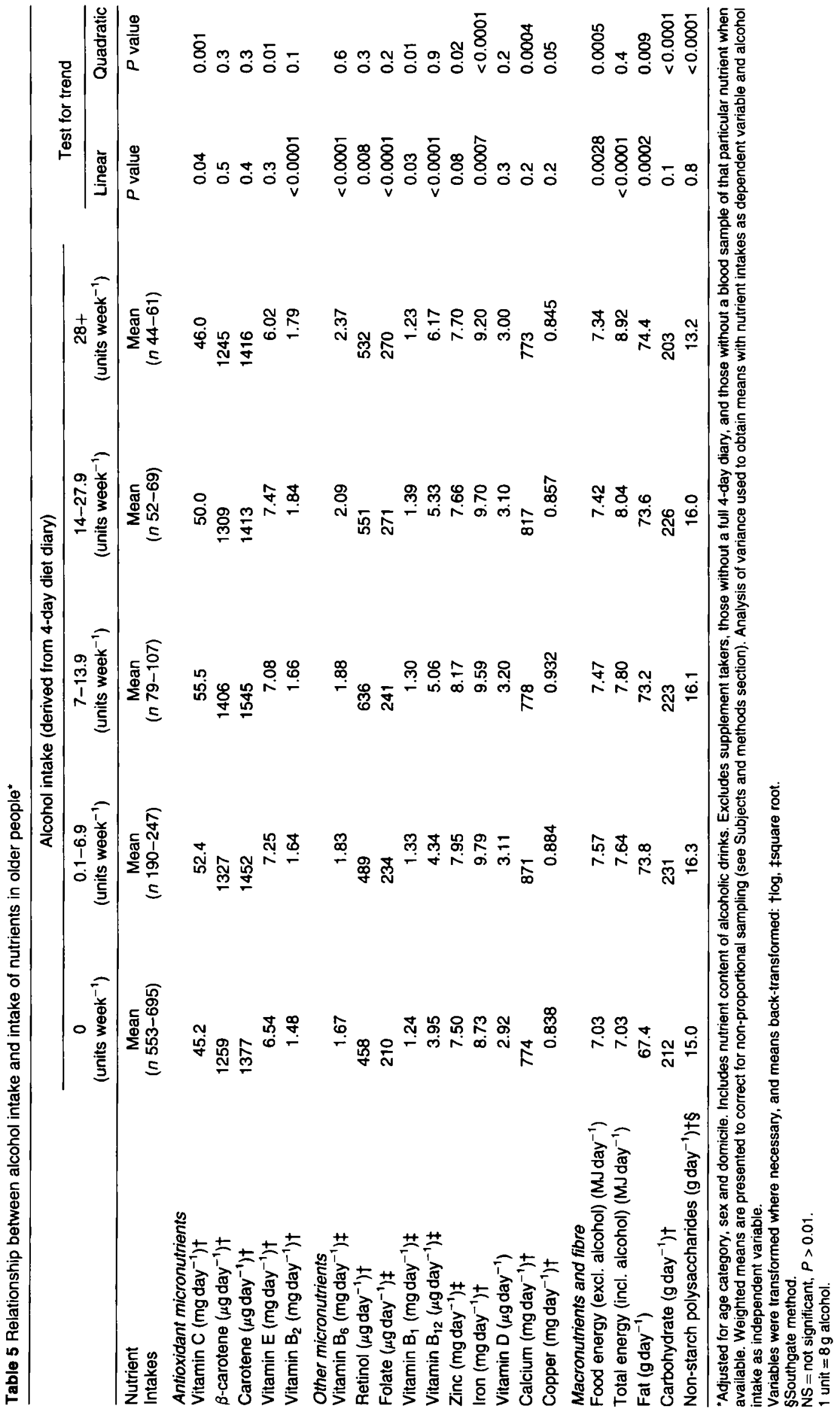




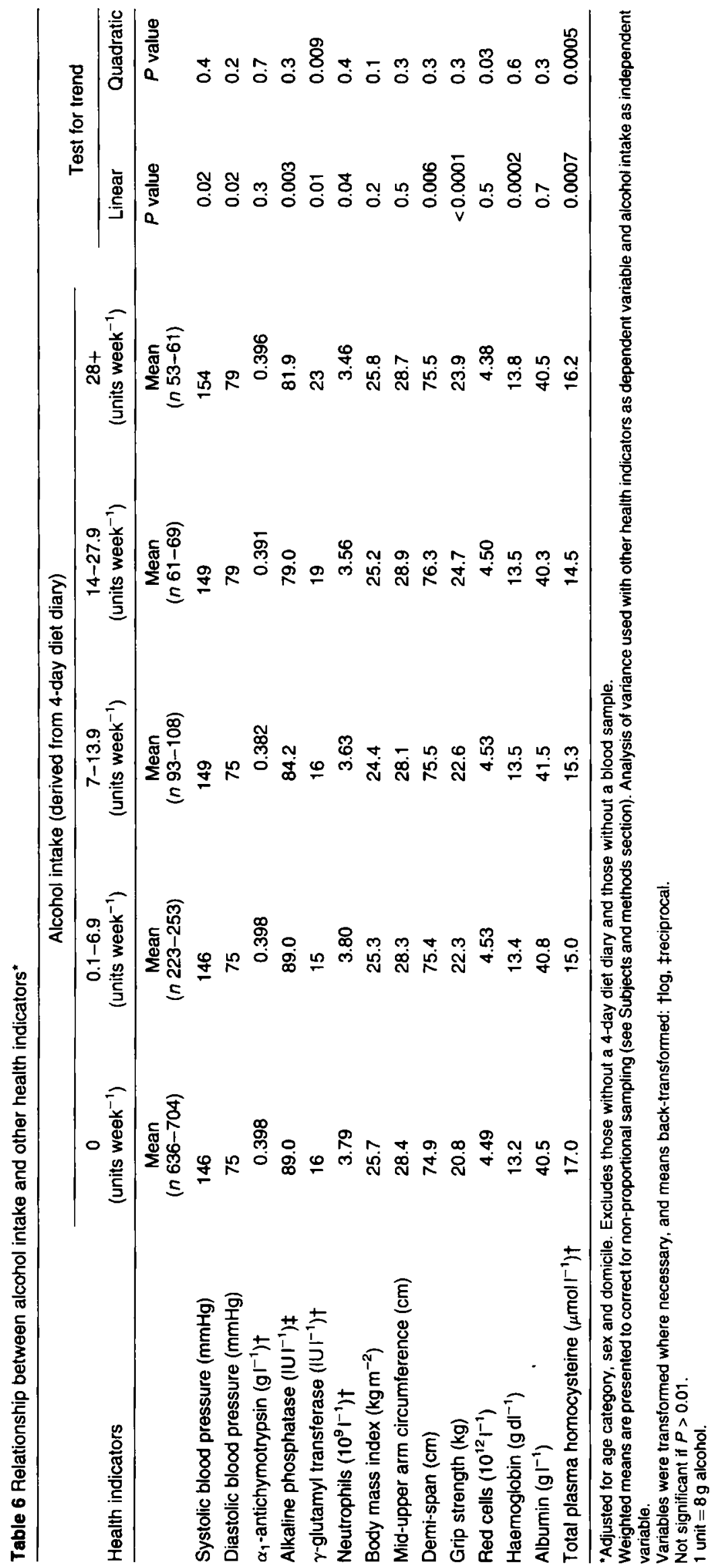


with intermediate alcohol intakes than non-drinkers or heavier drinkers. After further adjustment for other factors (B), alcohol was directly correlated with systolic blood pressure $(P=0.005)$, diastolic blood pressure $(P=0.01)$ and the significance of some associations fell outside the $1 \%$ level $(P \leq 0.01)$ : GGT $(P=0.03)$, tHcy $(P=0.02)$, demispan $(P=0.2)$ and haemoglobin $(P=0.1)$. Alcohol intake derived from the questionnaire was inversely associated with alkaline phosphatase only, after adjustment for age, sex, domicile and other factors (B) $(P=0.002)$.

\section{Discussion}

The present study has investigated the associations for nutrient intake and blood status indices with alcohol intake, estimated by two methods. The UK Department of Health has indicated that weekly (and therefore 4day) consumption levels may mask short term episodes of heavy drinking which often correlate with medical and social harm ${ }^{3}$, and this should be considered when interpreting the results.

The definition of a unit of alcohol varies in different countries and caution needs to be applied when comparing studies. For example, a unit of alcohol in the UK (and in the present study) is $8 \mathrm{~g}$ (the amount contained in half a pint of beer, or in a small glass of wine, or in a standard measure of spirits) ${ }^{3}$ whereas the US unit is defined as $12 \mathrm{~g}$ or $14 \mathrm{~g}^{21}$. Public health recommendations based on units of alcohol need therefore to be country specific, while comparisons between countries, and international public health recommendations, are better based on alcohol intake in grams.

Current sensible drinking advice in the UK recommends that men over 40 years and postmenopausal women may benefit from drinking 1 unit $(8 \mathrm{~g})$ alcohol a day, with the maximum health advantage, particularly for avoiding cardiovascular disease, lying between 1 and 2 units $(8-16 \mathrm{~g})$ alcohol a day ${ }^{3}$. This study has shown that the vast majority of older people in Britain appear to consume $<7$ units of alcohol per week, and less in the oldest age group, in females and those living in institutions.

It may be predicted that people would underestimate alcohol use in a 12-month recall questionnaire (perhaps more than in a 4-day diary), if it were perceived to be undesirable. However, there was little evidence from the present study that the 4-day diary revealed substantial alcohol use that was not revealed by the questionnaire.

The present study showed that after adjustment for age, sex, domicile and nutrient intake, light to moderate alcohol intakes were associated with higher blood concentrations of vitamin $\mathrm{C}$, lutein, $\beta$-cryptoxanthin, serum folate and calcium than abstainers or heavier drinkers, with heavy drinkers (28+ units week $^{-1}$ ) having the lowest concentrations of these nutrients. Alcohol intake was directly correlated with plasma lycopene, HDL-cholesterol, PLP and plasma retinol across the whole range of alcohol intake. Although some of these associations were attenuated after further adjustment for social class, cigarette smoking, self-reported health, grip strength and total energy intake, significance of these associations did not fall below the $5 \%$ level.

Other studies have reported lower levels of $\beta$-cryptoxanthin ${ }^{22}$ and plasma vitamin $\mathrm{C}^{23}$ in people with higher alcohol consumption. Malabsorption ${ }^{11}$, or oxidative stress from high alcohol intake and a subsequent increased utilization of antioxidants may be the cause of lower concentrations of $\beta$-cryptoxanthin ${ }^{22}$ and of other antioxidant nutrients, in heavier alcohol consumers

Folate deficiency is common in alcoholic patients $^{8,24,25}$ due to malabsorption, increased urinary folate excretion ${ }^{7,8}$, or alteration in cellular utilization and tissue distribution ${ }^{26}$, and the effect is doserelated $^{26}$. Accordingly, this study shows that despite lower folate intakes, blood concentrations of folate (both unadjusted and adjusted for folate intake) were higher in light than heavy alcohol users.

Other studies have found alcohol consumption to be associated with higher blood concentrations of iron $^{27}$, serum ferritin ${ }^{28}$ and retinol ${ }^{16,17,22}$. The mechanisms through which alcohol causes or enhances hyperferritinaemia are not clear ${ }^{28}$. The higher levels of retinol could be attributed to the effect of ethanol on retinol mobilization from the liver ${ }^{29}$. With reference to the higher concentrations of PLP (independent of vitamin $B_{6}$ intakes) in drinkers in this study it has been suggested that the bioavailability of vitamin $B_{6}$ from alcoholic beverages may be relatively high ${ }^{30}$.

Those who reported themselves to be in good health and less depressed were more likely to drink alcohol, particularly wine, in this study, although abstinence from alcohol was not found to be associated with long-standing illness, except diabetes. Other research has found that moderate drinking is associated with positive moods, a sense of well-being, and decreased morbidity ${ }^{3}$.

The explanation for why light to moderate alcohol intakes in this study are associated with higher blood concentrations of some antioxidants, independent of age, sex, domicile, nutrient intake, social class, selfreported health, cigarette smoking and grip strength, is not clear and requires further investigation, to determine whether these observations are due to the alcohol per se, other constituents in alcoholic drinks (such as flavonoids, antioxidants in wine) or whether moderate alcohol consumption is merely a proxy for good health and good nutrition. 
It has been observed that alcohol intakes in the range of 7-40 units of alcohol a week are associated with a $30-50 \%$ decreased risk of coronary heart disease, and this may be largely due to raised HDLcholesterol levels in the blood ${ }^{3}$. The present study showed a strong direct association between HDLcholesterol and alcohol intakes in older people, particularly from 7 units up to 28 or more units week ${ }^{-1}$.

Alcohol use was directly correlated with systolic and diastolic blood pressure in this study after adjustment for age, sex, domicile and other factors (B). Other studies have reported alcohol to be a strong predictor of hypertension ${ }^{31}$ and clinicians generally accept that for men the rise in blood pressure produced by 4 units day $^{-1}$ (about $6 \mathrm{mmHg}$ systolic and $4 \mathrm{mmHg}$ diastolic) would give cause for concern ${ }^{3}$.

Some studies have shown that light to moderate alcohol consumers do not substitute alcoholic beverages for food items, but consume alcohol in addition to normal food intakes ${ }^{32}$. However, others have shown that substitution in those drinking alcohol does occur ${ }^{33}$. It has been suggested that poorer people are more likely to replace food with alcohol ${ }^{10}$. The present study found energy from food was highest in those consuming light amounts of alcohol and lowest in those consuming either no alcohol or heavy amounts of alcohol. Light alcohol intake may have stimulated appetite in the present study, and some substitution of alcohol for foods may have occurred in those consuming heavier amounts of alcohol. Stimulation of appetite by low doses of alcohol has been reported elsewhere ${ }^{2}$. Despite the strong direct association between total energy and alcohol intake, there was no increase in body mass index (BMI) with alcohol intake. Some studies have reported lower BMIs in alcohol consumers than non-consumers ${ }^{33}$ and it has been suggested by some that energy from ethanol at high intakes may not be completely utilized ${ }^{10,33}$

Alcohol intake was directly correlated with intakes (including nutrient content of alcoholic drinks) of $\mathrm{B}$ vitamins $\left(B_{2}, B_{6}, B_{12}\right.$, folate), total energy intake and fat across the whole range of alcohol intakes after adjustment for age, sex, domicile and other factors (B). Those with intermediate alcohol intakes in this study had higher intakes of vitamins $C, E$ and $B_{1}$, iron, calcium, energy from food, carbohydrate and NSP than abstainers and heavy drinkers. Further adjustment for total energy intake attenuated the association between alcohol and intake of vitamin $B_{2}$ only. Other studies have shown lower intakes of vitamin $C^{5,32}$, calcium, vitamin $B_{1}$, carbohydrate $e^{32}$ and fibre ${ }^{34}$, and higher dietary fat ${ }^{5,34}$ with increasing alcohol consumption. The direct association between intakes of $\mathrm{B}$ vitamins and alcohol in this study was partially explained by the high content of certain $B$ vitamins in beer ${ }^{30}$.
Alcohol intake in this study was associated with consumption of meat products, pies and pasties; red meat and poultry; and fruit. No associations between other food groups and alcohol intake were observed. A recent study in Finland ${ }^{32}$ found lower intakes of a number of food groups with increased alcohol consumption, including the intakes of vegetables, fruit, meat, fish, wheat, other cereals and milk. Smoking is often associated with increased alcohol consumption $^{32,33,35-37}$ but the present study found no significant association between alcohol consumption and cigarette smoking after adjustment for age, sex and domicile.

In conclusion, this study of a representative sample of older people in mainland Britain showed that light to moderate alcohol intake is associated with higher intakes of some nutrients and higher concentrations of blood status indices for some micronutrients including antioxidants, independent of age, sex, domicile, nutrient intake, total energy intake, social class, selfreported health, cigarette smoking and grip strength. The explanation for the latter associations remains unclear and further research is recommended to investigate whether the alcohol per $s e$, or other constituents in alcoholic drinks, are responsible or whether moderate alcohol consumption is a merely a proxy for good health and nutrition. Heavier alcohol consumption (up to 28 or more units week ${ }^{-1}$ ) is associated both with beneficial and adverse effects on nutrient intakes, nutrient status and other health indicators.

\section{Acknowledgements}

The NDNS of people aged 65 years and over was funded jointly by MAFF and the Department of Health and conducted by Social and Community Planning Research (SCPR) in conjunction with the Medical Research Council's Dunn Nutrition Unit, the University of Newcastle, University College London and the University of Birmingham. The measurements of tHcy were performed by Dr MA Mansoor, Central Hospital in Rogaland, Stavanger, Norway. Measurements of PLP were performed by Dr K Pentieva. CM Walmsley was supported by MAFF.

\section{References}

1 Veenstra J, Ockhuizen $T$, Van de Pol $H$, Wedel $M$, Schaafsma G. Effects of a moderate dose of alcohol on blood lipids and lipoproteins postprandially and in the fasting state. Alcobol and Alcobolism 1990; 25: 371-7.

2 Dufour MC. Alcohol and the elderly. Health Prom. Dis. Prev. 1992; 8: 127-41.

3 Department of Health. Sensible Drinking. The Report of an Inter-Departmental Working Group. December 1995, Department of Health, London: HMSO.

4 Bjorneboe A, Bjorneboe G-Ea. Antioxidant status and 
alcohol-related diseases. Alcobol and Alcobolism 1993; 28 111-16

5 Lecomte E. Herbeth B, Pirollet P, et al. Effect of alcohol consumption on blood antioxidant nutrients and oxidative stress indicators. Am. J. Clin. Nutr. 1994; 60: 255-61.

6 Nordmann R. Alcohol and antioxidant systems. Alcobol and Alcoholism 1994; 29: 513-22.

7 Russell RM, Rosenberg IH, Wilson PD, et al. Increased urinary excretion and prolonged turnover time of folic acid during ethanol ingestion. Am. J. Clin. Nutr. 1983; 38: 64-70.

8 Bailey LB. The role of folate in human nutrition. Nutrition Today 1990; Sept/Oct: 12-19.

9 Sauberlich HE. Evaluation of folate nutrition in population groups. In: Folic Acid Metabolism in Health and Disease. New York: Wiley-Liss, 1990: 211-35.

10 Klein S, Iber FL. Alcoholism and associated malnutrition in the elderly. Nutrition 1991; 7: 75-9.

11 Ahmed S, Leo MA, Lieber CS. Interactions between alcohol and $\beta$-carotene in patients with alcoholic liver disease. $\mathrm{Am}$. J. Clin. Nutr. 1994; 60: 420-6.

12 Gloria L, Cravo M, Camilo ME, et al. Nutritional deficiences in chronic alcoholics: relation to dietary intake and alcohol consumption. Am. J. Gastroenterol. 1997; 92: 485-9.

13 Hendriks HFJ, Veenstra J, Velthuis-te Wierik EJM, Schaafsma $G$, Kluft $C$. Effect of moderate dose of alcohol with evening meal on fibrinolytic factors. BMJ 1994; 308: 1003-6.

14 Rolls BJ. Ageing and appetite. Nutr. Rev. 1992; 50: 422-6.

15 Department of Health. The Nutrition of Elderly People. Report on Health and Social Subjects, no. 43. London: HMSO, 1992.

16 Herbeth B, Chavance M, Musse N, Mejean L, Vernhes G. Dietary intake and other determinants of blood vitamins in an elderly population. Eur. J. Clin. Nutr. 1989; 43: 175-86.

17 Hercberg S, Preziosi P, Galan P, et al. Vitamin status of a healthy French population: dietary intakes and biochemical markers. Int. J. Vit. Nutr. Res. 1994; 64: 220-32.

18 Heseker $\mathrm{H}$, Schneider $\mathrm{R}$. Requirement and supply of vitamin $\mathrm{C}, \mathrm{E}$ and $\beta$-carotene for elderly men and women. Eur.J. Clin. Nutr. 1994; 48: 118-27.

19 Finch S, Doyle W, Lowe C, et al. National Diet and Nutrition Survey: People Aged 65 Years and Over, vol. 1: Report of the Diet and Nutrition Survey. London: SO, 1998 (in press)

20 Hughes JM, Smithers G, Gay C, et al. The British National Diet and Nutrition Survey of people aged 65 years or over: protocol and feasibility study. Proc. Nutr. Soc. 1995; 54: 631-43.

21 Doll R. One for the heart. Clinical review. BMJ 1997; 315 1664-8.

22 Albanes D, Virtamo J, Taylor PR, Rautalahti M, Pietinen P, Heinonen OP. Effects of supplemental $\beta$-carotene, cigarette smoking, and alcohol consumption on serum carotenoids in the $\alpha$-tocopherol, $\beta$-carotene Cancer Prevention Study. Am. J. Clin. Nutr. 1997; 66: 366-72.

23 Fazio V, Flint DM, Wahlqvist ML. Acute effects of alcohol on plasma ascorbic acid in healthy subjects. Am.J. Clin. Nutr. 1981; 34: 2394-6.

24 Halsted $\mathrm{CH}$. Folate deficiency in alcoholism. Am. J. Clin. Nutr. 1980; 33: 2736-40.

25 Bonjour JP. Vitamins and alcoholism II. Folate and vitamin B 12 . Int.J. Vit. Nutr. Res. 1980; 50: 96-121

26 McMartin KE, Collins TD, Shiao CQ, Vidrine L, Redetzki HM. Study of dose-dependence and urinary folate excretion produced by ethanol in humans and rats. Alcobolism: Clin. Exp. Res. 1986; 10: 419-24.

27 Cook CCH, Walden RJ, Graham BR, Gillham C, Davies S Prichard BNC. Trace element and vitamin deficiency in alcoholic and control subjects. Alcobol and Alcobolism 1991; 26: 541-8.

28 Charlton KE, Kruger $M$, Labadarios $D$, Wolmarans $P$, Aronson I. Iron, folate and vitamin $B_{12}$ status of an elderly South African population. Eur. J. Clin. Nutr. 1997; 51 $424-30$.

29 Simonetti P, Brusamolino A, Pellegrini N, et al. Evaluation of the effect of alcohol consumption on erythrocyte lipids and vitamins in a healthy population. Alcobolism. Clin. Exp. Res. 1995; 19: 517-22.

30 Lowik MRH, van Poppel $G$, Wedel $M$, van den Berg $H$ Schrijver $\mathrm{J}$. Dependence of vitamin $\mathrm{B}_{6}$ status assessment on alcohol intake among elderly men and women (Dutch Nutrition Surveillance System). Am. Inst. Nutr. $1990 ; 1344-51$.

31 Dwyer JH, Li L, Dwyer KM, Curtin LR, Feinleib M. Dietary calcium, alcohol, and incidence of treated hypertension in the NHANES I. Epidemiologic follow-up study. Am. $J$. Epidemiol. 1996, 144: 828-38.

32 Mannisto S, Pietinen P, Haukka J, Ovaskainen M-L, Albanes $D$, Virtamo J. Reported alcohol intake, diet and body mass index in male smokers. Eur. J. Clin. Nutr. 1996; 50: 239-45.

33 Mannisto S, Uusitalo K, Fogelholm M, Pietinen P. Alcohol beverage drinking, diet and body mass index in a crosssectional survey. Eur. J. Clin. Nutr. 1997; 51 : 326-32.

34 Rimm E, Colditz G. Smoking, alcohol, and plasma levels of carotenes and vitamin E. Ann. NY Acad. Sci. 1993; 686: 323-34.

35 Schectman G, Byrd JC, Gruchow HW. The influence of smoking on vitamin C status in adults. Am. J. Public Health 1989; 79: $158-62$.

36 Zondervan KT, Ocke MC, Smit HA, Seidell JC. Do dietary and supplementary intakes of antioxidants differ with smoking status. Int. J. Epidemiol. 1996; 25: 70-9.

37 Ross MA, Crosely LK, Brown KM, et al. Plasma concentrations of carotenoids and antioxidant vitamins in Scottish males: influence of smoking. Eur. J. Clin. Nutr. 1995; 49: 861-5. 